(b) It introduces into the eigenfunctions admixtures of wave functions belonging to other eigenvalues of the exchange energy, thereby allowing weak transitions to states excluded by the original selection rule. This is most marked for states differing only slightly in exchange energy, and gives rise to a 'tail' on the absorption curve, falling off roughly inversely as the square of the distance from resonance. It may also give rise to enhanced absorption at low frequencies and at twice the resonant frequency. This effect increases with magnetic field.

(c) The coupling with the oscillating field being unsymmetrical with respect to the variables of the two types of ion, transitions can be induced to states of different exchange energy. This effect is similar to (b), except that it shows no marked preference for small differences of exchange energy and is roughly independent of $H$. It gives a background of nonresonant absorption.

A straightforward application of second-order perturbation methods (the first-order effect is zero) leads to the following expression for the dependence of effect $(a)$ on orientation and frequency. Using a notation in which $l_{1}, l_{2}, l_{3}$ are the cosines of the angles between the magnetic field and the principal magnetic axes, and $g_{1}, g_{2}, g_{3}$ are the principal $g$-values, the breadth $\Delta H_{\text {ex }}$ due to exchange, for fixed frequency $\nu$, is given by

$$
\begin{aligned}
& \Delta H_{\mathrm{ex}}= \\
& \frac{\left[\begin{array}{l}
4\left(g_{2}+g_{3}\right)^{4} l_{2}{ }^{4} l_{3}{ }^{4} \\
-4 A(\nu)\left(g_{2}+g_{3}\right)^{2} l_{2}{ }^{2} l_{3}{ }^{2}\left\{g_{1}{ }^{2} l_{1}{ }^{2}\left(l_{2}{ }^{2}+l_{3}{ }^{2}\right)+\left(g_{2} l_{2}{ }^{2}-g_{3} l_{3}{ }^{2}\right)^{2}\right\} \\
+B(\nu)\left\{g_{1}{ }^{2} l_{1}{ }^{2}\left(l_{2}{ }^{2}+l_{3}{ }^{2}\right)+\left(g_{2} l_{2}{ }^{2}-g_{3} l_{3}{ }^{2}\right)^{2}\right\}^{2}
\end{array}\right.}{\left[g_{1}{ }^{2} l_{1}{ }^{2}+g_{2}{ }^{2} l_{2}{ }^{2}+g_{3}{ }^{2} l_{3}{ }^{2}\right]^{5 / 2}}
\end{aligned}
$$

where $C$ is a constant depending on the magnitude of the exchange energy but independent of orientation, temperature and frequency, and $A(v), B(v)$ are independent of angle and temperature, and tend to 1 in the limit of low frequencies (small compared with the exchange frequency, that is, about $0.15 \mathrm{~cm}^{-1}$ ).

The breadth is to be interpreted as a root mean square deviation from the centre of resonance. In comparing with experiment it should be noted that it refers to the Gaussian part of the peak and ignores the tail. The formula will vary in a more complicated manner with orientation if the individual ions are not uniaxial, but will involve the frequency in the same way.

If one wishes to include the effect of magnetic interaction one may, as a rough estimate, make the assumption that the square of the total breadth is the sum of squares of partial breadths

$$
(\Delta H)^{2}=\left(\Delta H_{\mathrm{ex}}\right)^{2}+\left(\Delta H_{\mathrm{magn}}\right)^{2}
$$

and suppose that $\Delta H_{\text {magn }}$ is not very sensitive to orientation.

It is to be emphasized that (2) is valid only for frequencies small compared with $1 \mathrm{~cm}^{-1}$, and provided $\mu H \ll k T$.

\section{Clarendon Laboratory, Oxford.}

$$
\text { M. H. L. Pryce }
$$

$$
\text { July } 26 .
$$

1 De Klerk, Physica, 12, 513 (1946).

${ }^{2}$ Gorter and Van Vleck, Phys. Rev., 72, 1128 (1947).

3 Bloembergen, Pound and Purcell, Phys. Rev., 71, 466 (1947).

- Bagguley and Griffiths, Nature (preceding communication).

s Polder, Physica, 9, 709 (1942).

- Krishnan and Mookherji, Phys. Rev., 50, 860 (1936); 54, 533 (1938): 54, 841 (1938).

'Opechowski, Physica, 14, 237 (1948). tiction

\section{ROYAL OBSERVATORY, GREENWICH}

$T$ HE Report of the Astronomer Royal to the Board of Visitors of the Royal Observatory, Greenwich, read on the occasion of the annual visitation of the Observatory on June 5, covers the period May 1, 1947-April 30, 1948, and the state of the Observatory mentioned in various parts refers to the latter date. Although a large amount of the report deals with the usual routine work, certain portions are concerned with the grave difficulties that have arisen in the post-war period, which may not be completely overcome for several years. This short synopsis of the report will deal chiefly with these difficulties.

Stage 1, as it is called, of the move to Herstmonceux was arranged to take place in August and was intended to include the Nautical Almanac Office, the Chronometer Depot, the Magnetic and Meteorological Department, and the secretariat; the Solar Department will wait until the new Solar Building is ready. Even under normal conditions, the removal would have entailed considerable disorganisation to the work of the Observatory; but this disorganisation is greatly increased by the difficulties in the present conditions. No provision has been made in the estimates for the current financial year for the completion of Stage 1 of the move, and the output of the Observatory is necessarily curtailed with every month of delay. As might be expected, some of the instruments are not in use, and there has been great disappointment regarding the performance of one in particular, about which something will be said later. The 28-in. and 26-in. domes are in a bad state of repair, and it is doubtful whether either is suitable for transfer to Herstmonceux; until this point has been settled, no work on them will be undertaken. The 28-in. and 26-in. telescopes were dismounted in the autumn of 1947, and the former, with the exception of the lens and some minor parts, was transported by road to Herstmonceux, where it is stored in a brick hut. The necessary parts of the drive have been sent to Sir Howard Grubb, Parsons and Co. for alteration to a synchronous motor drive. Practically all the 26-in. telescope has been sent to the same firm, as it was decided that roller bearings in both axes should be inserted. The objectives of these telescopes are still in good condition and remain for the present at Greenwich.

The mirrors of the 36-in. Yapp reflector were aluminized in May 1946; but the instrument remained unused until November. In December the 36-in. mirror was given a light alcohol wash and was then found to be in poor condition. It was used until March 1948, when it was discovered that several areas each about two inches across had lost all their coating, and after a fresh wash, the reflexion coefficient was estimated to be as low as about 30 per cent. The mirror was cleaned and silvered; but surface blemishes remained visible on the glass even after silvering and it is not certain how much of this can be attributed to poor aluminizing and how much to the deposit of chemically active dirt. It is interesting to know that the Cassegrain mirror was, and still is, in good condition, and the contrast between the two mirrors confirms what has been already known from other pairs of aluminized mirrors, one 
facing up and one down, in the conditions which prevail at Greenwich. The expense and delay involved in sending the mirror away to be aluminized are not warranted while the mirror remains at Greenwich; but as the spectrograph is primarily for use in the ultra-violet region, the effectiveness of the telescope is very much reduced. When an aluminizing plant capable of handling the 36 -in. reflector is installed at Herstmonceux, this state of affairs will be remedied, and this is just one among many reasons for accelerating the move to Herstmonceux.

If there be any who expect a greatly increased output from the Observatory in the near future, they will be disillusioned when they read the present report. It is emphasized that the situation which will prevail from the end of Stage 1 until completion of the move will be very disadvantageous in many ways, not least of which will be the marked excess of observers over instruments at Herstmonceux and Abinger. Many valuable years of potential training have been lost already; old observers are retiring and a new and inexperienced staff is increasing. Even if the dismemberment of the Greenwich Observatory could be effected immediately, much ground would have to be made up, and the longer the delay in unification the more serious will be the effects on the standards of observational skill. This is a matter which requires immediate consideration from the authorities, and another matter is of equal or even greater urgency.

While the hostel arrangements will cater reasonably well for the unmarried staff, no arrangements have yet been made for marxied men. It is almost certain that most married members of the staff, some of whom have had as much as six years war experience, will be faced with a period of at least a year's separation from their families. The continuance of a similar situation through all the later stages of the move must cause grave dissatisfaction and discouragement, and would have an adverse effect on the smooth running of the Observatory. The Astronomer Royal has proposed to the Admiralty that houses should be provided for the senior observing staff, and the necessity for such accommodation, which is fairly normal for observatories, is obvious to everyone who knows that the Observa. tory at Herstmonceux is in an isolated area. Such provision on the edge of the Observatory property would greatly facilitate the night duties of the observing staff and also reduce the loss of observing hours. It is pointed out that it may be difficult to fill the additional vacancies for senior scientific workers which will occur later, because it is highly probable that many married men will refuse an offer which implies separation from their families. In the words of the Astronomer Royal : "There is evidently a grave risk that the increase in complement, which has been approved in principle, may be nullified in practice through an unrealistic approach to the housing problem, quite apart from the general hardship which is, as yet, the only prospect for existing married staff of the later stages".

The report devotes a little more than a page to the Isaac Newton Observatory which will be erected in the grounds at Herstmonceux. Among the types of observation to be undertaken with the 100 -in. telescope are the following: stellar spectroscopy and spectrophotometry, star counts, galactic structure, the investigation of the distribution and extent of obscuring material, spectral classification with a slitless telescope, the determination of radial veloci- ties, and observations of extra-galactic nebulæ. Details regarding optical design are still under consideration, and information will be obtained about the system of control which has been developed for the 200 -in. telescope, and also on the methods of driving and control of other large telescopes. Messrs. Pilkington Bros., Ltd., have carried out investigations on the manufacture of large glass mirror disks, and it is proposed to make the 100 -in. disk of 'Pyrex' glass and to cast it as a solid disk, the lower face being given a concave curvature of approximately the required amount. It is expected that the casting will be undertalken about March 1949.

The magnetic elements as determined at Abinger for 1947 and also the three preceding years are as follows :

\begin{tabular}{|c|c|c|c|c|c|}
\hline & $\begin{array}{l}\text { Declination } \\
\text { (West) }\end{array}$ & $\underset{\text { intensity }}{\text { Horizontal }}$ & $\begin{array}{c}\text { Vertical } \\
\text { intensity }\end{array}$ & Incl & inati \\
\hline 4 & $10^{\circ} 07 \cdot 8^{\prime}$ & 0.18566 & 0.43189 & $66^{\circ}$ & \\
\hline 12 & $9 \quad 59.5$ & 0.18573 & 0.43207 & 66 & 44 . \\
\hline$O A$ & $51 \cdot 1$ & 0.18569 & 0.43235 & & \\
\hline 194 & $943 \cdot 1$ & 0.18577 & 0.43246 & 66 & 45 . \\
\hline
\end{tabular}

At the time of writing the above summary of the Astronomer Royal's Report, it was known that there had been a delay in carrying out Stage 1 of the movement to Herstmonceux.

\section{RESISTANCE TO SULPHADIAZINE AND 'PALUDRINE' IN THE MALARIA PARASITE OF THE FOWL ( $P$. gallinaceum) By DR. ANN BISHOP}

ELSPETH W. MCCONNACHIE

Molteno Institute, University of Cambridge

\section{Acquired Resistance to Sulphadiazine}

$\mathrm{R}$ ECENTLY Bishop and Birkett ${ }^{1}$, and Williamson, Bertram and Lourie ${ }^{2}$, working independently, have described the development of resistance to the antimalarial drug 'Paludrine' in infections of Plasmodium gallinaceum in young chicks. This resistance developed rapidly and increased forty-fold within about four and a half months. With sulphadiazine and quinine, however, Williamson and his co-workers obtained no acquired resistance in $P$. gallinaceum, though the periods of drug treatment extended over two and a half years. Since the publication of their results, Knoppers ${ }^{3}$ has described a twofold increase in resistance to quinine in the same species of avian malaria. Bishop and Birkett found that resistance to sulphadiazine could be produced in $P$. gallinaceum in young chicks by methods similar to those with which they had induced resistance to 'Paludrine', though its development was relatively slow and the degree of resistance obtained was not great.

As a result of further treatment with gradually increasing doses of sulphadiazine, we have now obtained a strain of $P$. gallinaceum in young chicks in which the resistance to the drug is thirty-two times greater than that of the untreated strain. Whereas the minimum dose of drug which produces an adverse effect upon the growth-rate of the normal strain, when given twice daily on successive days, is $0.625 \mathrm{mgm} . / 20 \mathrm{gm}$. of body-weight, in the resistant strain growth of the parasites occurs in birds receiving 